\title{
Pediatric Traumatic Brain Injury: an Update on Management
}

\author{
Michelle T. Gardner $^{1}$ • Alia Marie Iqbal O'Meara ${ }^{1,2}$ • Nikki Miller Ferguson ${ }^{1}$
}

Published online: 6 October 2017

(C) Springer Science+Business Media, LLC 2017

\begin{abstract}
Purpose of Review This article reviews the pathophysiology, evaluation, and management of pediatric TBI, as well as highlights recent updates in the literature. Traumatic brain injury (TBI) is a leading cause of death and permanent disability worldwide, and its prevalence presents a significant public health concern. Since the second edition of the Guidelines for the Acute Medical Management of Severe Traumatic Brain Injury in Infants, Children, and Adolescents was released, several recent studies have broadened our understanding of pediatric TBI.

Recent Findings While there remains a paucity of high-level evidence on which to base precise consensus guidelines for care in pediatric patients, recent studies have addressed therapeutic hypothermia, pharmacologic treatment of elevated intracranial pressure, and abusive head trauma.

Summary Understanding the pathophysiology and treatment strategies for pediatric TBI, while preventing secondary insults, remains a cornerstone for improving patient outcomes.
\end{abstract}

Keywords Traumatic brain injury · Intracranial hypertension . Decompressive craniectomy $\cdot$ Hypothermia $\cdot$ Posttraumatic seizures $\cdot$ Hyperosmolar therapy

This article is part of the Topical Collection on Intensive Care Medicine

Nikki Miller Ferguson

nikki.millerferguson@vcuhealth.org

1 Department of Pediatrics, Virginia Commonwealth University, 1008 East Clay St, B-009, P.O. Box 980530, Richmond, VA 23298, USA

2 Department of Pediatrics, Uniformed Services University of the Health Sciences, Bethesda, MD, USA

\section{Introduction}

Despite increases in prevention programs, traumatic brain injury (TBI) remains a leading cause of morbidity and mortality in children worldwide, and is a major public health concern posing a significant burden on the healthcare system. Critical brain development occurs during infancy and childhood, and children may be more selectively vulnerable to TBI. Sustaining a brain injury in early childhood can delay or interrupt foundational neurodevelopment [1]. The Guidelines for the Acute Medical Management of Severe Traumatic Brain Injury in Infants, Children, and Adolescents, now in its second edition, have become a cornerstone for systematically evaluating treatment strategies with the additional aim of identifying high yield research questions to drive the field forward [2]. Despite extensive gains in our understanding of the molecular basis of TBI, there remains no specific "magic bullet" therapy to improve outcomes. There is a striking paucity of high-level evidence for specific goals in the management of pediatric TBI, though important studies continue to demonstrate that meticulous attention to resuscitation and supportive care, with expedient surgical intervention when warranted, is paramount [2]. Table 1 showcases articles published within the past 5 years that have expanded our knowledge of pediatric TBI and will be referenced throughout this review article.

\section{Epidemiology}

According to the latest Center for Disease Control (CDC) report, in 2013 there were a total of 2.8 million TBI-related emergency department (ED) visits, hospitalizations, and deaths in the USA involving all age groups. Of those, a total 
Table 1 Notable pediatric TBI articles published within the previous 5 years

\begin{tabular}{|c|c|c|c|}
\hline Study & Authors & Study description & Conclusions \\
\hline $\begin{array}{l}\text { Abusive head trauma and mortality_an } \\
\text { analysis from an international comparative } \\
\text { effectiveness study of children with severe } \\
\text { traumatic brain injury }\end{array}$ & $\begin{array}{l}\text { Miller Ferguson } \\
\text { et al. } 2017\end{array}$ & $\begin{array}{l}\text { Design: comparative effectiveness study using } \\
\text { an observational, cohort study design. } \\
N=200 \text { patients from ADAPT trial } \\
\text { Purpose: determine the impact of abusive head } \\
\text { trauma on mortality; identify factors } \\
\text { differentiating children with abusive head } \\
\text { trauma causing traumatic brain injury } \\
\text { compared to other mechanisms of brain } \\
\text { injury. }\end{array}$ & $\begin{array}{l}\text { Female predominance was seen in } \\
\text { AHT cohort, no difference in } \\
\text { mortality between AHT and } \\
\text { accidental TBI cohorts } \\
\text { Patients with AHT may benefit } \\
\text { from ICP directed therapy }\end{array}$ \\
\hline $\begin{array}{l}\text { Effectiveness of pharmacologic therapies for } \\
\text { intracranial hypertension in children with } \\
\text { severe traumatic brain injury-results from } \\
\text { an automated data collection system } \\
\text { time-synched to drug administration }\end{array}$ & Shein et al. 2016 & $\begin{array}{l}\text { Design: prospective, observational study } \\
N=16 \text { children, total of } 196 \text { doses of fentanyl, } \\
\text { hypertonic saline, mannitol, and } \\
\text { pentobarbital } \\
\text { Purpose: describe cerebral hemodynamic } \\
\text { effects of medications used to treat } \\
\text { intracranial hypertension }\end{array}$ & $\begin{array}{l}\text { Hypertonic saline resulted in the } \\
\text { most rapid resolution of ICP, as } \\
\text { well as increased CPP } \\
\text { Fentanyl was associated with } \\
\text { acutely decreased CPP and } \\
\text { highest treatment failure rate }\end{array}$ \\
\hline $\begin{array}{l}\text { Age-specific cerebral perfusion pressure } \\
\text { thresholds and survival in children and } \\
\text { adolescents with severe traumatic brain } \\
\text { injury }\end{array}$ & Allen et al. 2014 & $\begin{array}{l}\text { Design: prospective, observational cohort } \\
\text { study } N=317<17 \text { years } \\
\text { Purpose: determine age-specific thresholds of } \\
\text { CPP on short-term survival }\end{array}$ & $\begin{array}{l}\text { Found age specific CPP thresholds } \\
0-5 \text { years }>40 \mathrm{mmHg} \\
6-17 \text { years }>50 \mathrm{mmHg} \\
\text { Increased ICP significantly related } \\
\text { to all decreased CPP events in all } \\
\text { ages }\end{array}$ \\
\hline $\begin{array}{l}\text { Comparison of hypothermia and } \\
\text { normothermia after severe traumatic brain } \\
\text { injury in children (Cool Kids): a phase } 3 \text {, } \\
\text { randomized controlled trial }\end{array}$ & $\begin{array}{l}\text { Adelson et al. } \\
2013\end{array}$ & $\begin{array}{l}\text { Design: phase } 3 \text {, multicenter, multinational } \\
\text { randomized controlled trial } \\
N=79 \text { patients total } \\
\text { Purpose: evaluate effect of therapeutic } \\
\text { hypothermia for } 48-72 \mathrm{~h} \text { with slow } \\
\text { rewarming on mortality }\end{array}$ & $\begin{array}{l}\text { No reduction in mortality or } \\
\text { improvement in global functional } \\
\text { outcome }\end{array}$ \\
\hline
\end{tabular}

$A D A P T$ acute decisions and approaches in pediatric TBI, $A H T$ abusive head trauma, $I C P$ intracranial pressure, $C P P$ cerebral perfusion pressure

of 1.1 million TBI-related ED visits, hospitalizations, and deaths involved patients aged 0-24 years [3]. Approximately 56,000 total deaths resulted from TBI, including almost 8000 deaths in the 0-24-year age group [3]. In children less than 4 years of age, abusive head trauma (AHT), accounts for a large portion of TBI-related admission and death, while the leading cause of TBI-related death in older pediatric patients is motor-vehicle collision [3, 4]. Males have been shown to have a higher predominance of TBI compared with females; however, a recent multicenter study of pediatric TBI patients by Miller Ferguson et al. highlights a female predominance in their AHT cohort, differing from most previous reports [5•].

\section{Pathophysiology}

Primary tissue disruption in TBI is mechanical in nature, including contusion after direct impact; shear stress from acceleration, deceleration, and rotational force; and laceration from depressed skull fractures or penetrating injury. Host response mechanisms create a secondary brain injury milieu, occur in the minutes and hours after insult, and can persist for days: ischemia, reperfusion, antioxidant depletion, excitotoxicity, energy failure, cytokine release, microglial activation, blood brain barrier, and vascular permeability. These mechanisms activate inflammatory and apoptotic cell death cascades and contribute to injury propagation and cerebral edema. Another important concept in TBI is axonal injury, primarily due to physical shearing of axons, but also resulting from Wallerian degeneration after neuronal injury or loss. The injured brain is especially vulnerable to hypoxia and hypotension, and expedient resuscitation is crucial in avoiding these secondary insults in the prehospital environment, emergency department, and pediatric intensive care unit. It has long been known that episodes of hypoxia or hypotension can significantly impact mortality after TBI, and a recent study by Kannan et al. revealed that timely resuscitation of these factors in early care was associated with lower in-hospital mortality in pediatric TBI patients [6-8].

Cerebral edema is a frequent complication after pediatric traumatic brain injury, which can arise from a combination of cytotoxic swelling of injured neurons and glia, vasogenic edema from a permeable blood brain barrier, and osmolar swelling of interstitial spaces as well as the cellular network. 
Cerebral edema, whether global or focal, peaks $24-72 \mathrm{~h}$ after injury and exacerbates injury by limiting blood flow and substrate diffusion, and thereby oxygen and glucose delivery. It is imperative to anticipate worsening cerebral edema in patients with severe TBI, and if unimpeded, may ultimately lead to herniation and a lethal restriction of all cerebral circulation. The Monro-Kellie doctrine states that the cranium is a fixed vault comprised of brain parenchyma, intravascular blood, and cerebrospinal fluid (CSF). An increase in one component mandates a decrease in another. This concept guides one of the major management strategies in severe TBI, controlling ICP. This is accomplished with pharmacologic therapies, CSF diversion, blood pressure augmentation, carefully controlled mild hyperventilation, and decompressive craniectomy, which will be discussed separately.

\section{Diagnosis and Neuroimaging}

TBI has classically been stratified according to level of consciousness with Glascow Coma Scale (GCS) score as the standard assessment tool. GCS $\geq 13$ defines mild TBI, GSC 9-12 defines moderate TBI, and GCS $\leq 8$ defines severe TBI.

Neuroimaging after injury is important in triaging patients and identifying intracranial pathology warranting neurosurgical intervention, e.g., extra-axial hemorrhage or depressed skull fracture. Computed tomography (CT) has been the primary modality used since it is quick and readily available in developed countries. While magnetic resonance imaging (MRI) may be more sensitive than CT for evaluating intracranial pathology, there is no evidence to support routine use of MRI in immediate management [2]. Once the patient is stabilized, MRI is often obtained to further delineate the extent of intracranial injuries and aid in prognostication, and is particularly useful in elucidating the contribution of diffuse axonal injury. Additionally, there is no evidence to support routine repeat head CT $>24 \mathrm{~h}$ after initial imaging in the absence of clinical change [2].

\section{Management}

Initial resuscitation and correction of respiratory and circulatory failure takes precedence when treating patients with TBI. Once the patient has been adequately resuscitated, therapies should focus on minimizing secondary injuries, reducing intracranial hypertension, and maintaining appropriate cerebral perfusion pressure (CPP). ICP monitoring, which may be considered in patients with severe TBI, is generally achieved with an intraparenchymal monitor or external ventricular drain (EVD), with the latter having the added benefit of therapeutically draining CSF for increased ICP (discussed separately) [2]. Management strategies include CSF diversion, sedation, analgesics, neuromuscular blockade, hyperosmolar therapy, blood pressure augmentation, mild hyperventilation, and decompressive craniectomy. A tiered approach for treatment of intracranial hypertension is listed in Table 2. The details of each treatment option will be discussed separately.

\section{Intracranial Pressure and Cerebral Perfusion Pressure Management}

One of the most vital components of TBI management is maintaining "appropriate" ICP and CPP (CPP = mean arterial pressure $[\mathrm{MAP}]-\mathrm{ICP})$. In the injured brain, cerebral blood flow autoregulation may be disrupted, and as such, reductions in CPP result in cerebral ischemia. There is evidence that sustained drops in CPP are associated with poor outcomes in patients following severe TBI $[9,10]$. A confounder particular to pediatric TBI is that "one size does not fit all". Blood pressure parameters vary widely between infancy and adolescence, and a recent study reported agespecific CPP thresholds associated with poor outcome [11•]. However, the current brain trauma foundation guidelines could only recommend that a minimum CPP of $40 \mathrm{mmHg}$ may be considered in severe TBI [2]. A slightly increased minimum CPP level of $45 \mathrm{mmHg}$ was recently reported, where the number of hours spent below this threshold was associated with worse outcomes [12]. Similarly, ICP treatment thresholds are also not well established in children, but sustained increases in ICP $\geq 20 \mathrm{mmHg}$ are associated with poor outcomes [12-15]. As such, it is recommended to treat ICP above a threshold of $20 \mathrm{mmHg}$ [2].

\section{Cerebrospinal Fluid Diversion}

Patients with intracranial hypertension can benefit from drainage of CSF, as it has been shown to lower ICP by decreasing total intracranial fluid volume [16-18]. Diversion of CSF is accomplished through an EVD, which is placed through a burr hole in the skull and into the ventricular system. This has its own inherent complications, including bleeding, malpositioning, and infection. Although CSF drainage can be achieved intermittently or continuously, continuous drainage has been shown to reduce ICP more effectively [17-19]. While this decision is often provider/institutional specific, we believe that continuous drainage of CSF provides a greater benefit to patients as it is less reactionary. Treatment of intracranial hypertension begins as soon as ICP starts to increase, and continuous drainage may potentially lessen the need for other ICP-directed therapy. An important potential benefit of CSF diversion is the ability to readily collect CSF samples for analysis. While currently undertaken in only a few academic centers and still in its infancy in 
Table 2 Tiered approach for treatment of intracranial hypertension

\begin{tabular}{lll}
\hline Tier 1 therapy & Tier 2 therapy & Tier 3 therapy \\
\hline Head of bed $30^{\circ}$ & Hyperosmolar therapy & Barbiturate coma \\
Sedation/analgesia & CSF diversion & Decompressive craniectomy \\
Neuromuscular blockade & Augmentation of BP/CPP & Mild hypothermia \\
Mild hyperventilation & & \\
\hline
\end{tabular}

$C S F$ cerebrospinal fluid, $B P$ blood pressure, $C P P$ cerebral perfusion pressure terms of discerning injury severity and titrating interventions, CSF biomarker measurement is yet another possible tool and one might expect future studies to demonstrate its utility in routine clinical practice $[17,20]$.

\section{Pharmacologic Therapies}

Analgesics, sedatives, and neuromuscular blocking agents are mainstays in the pediatric intensive care unit for patient safety and comfort with medical interventions and devices such as mechanical ventilators, and they are routinely used to treat elevated ICP. Opioids blunt transmission of painful stimuli to the central nervous system, and have useful sedative sideeffects. Sedative agents reduce cerebral activity and metabolic demand thus decreasing blood flow and ultimately ICP. The gamma-aminobutyric acid (GABA) agonist sedatives dexmedetomidine, benzodiazepines, and barbiturates shift the neurotransmitter balance toward inhibition and sleep. Though use in pediatrics is quite controversial and the FDA has issued a warning for the pediatric population due to the association with infusion-related lactic acidosis, the mixed GABA agonist and N-methyl-D-aspartate (NMDA) antagonist propofol is a highly effective anesthetic sedative with the added benefit of a very short half-life, facilitating clinical neurologic examination [2]. Historically, ketamine, an NMDA antagonist, has been avoided for use in pediatric TBI as it increases catecholamine release, causing an increase in systemic vascular resistance, suggesting that the pharmacodynamics of ketamine may cause increased cerebral blood flow and ICP. However, recent studies and reviews in adult and pediatric TBI suggest that ketamine does not increase ICP and possibly increases CPP, though further studies are recommended [21-23]. Neuromuscular blocking agents are common adjuncts in the management of intracranial hypertension in order to reduce metabolic demands beyond sedation and prevent shivering when treating hyperthermia with external cooling measures. Caution must be exercised when administering paralytics; however, as they mask clinical seizures, it is of utmost importance to continuously monitor patients with electroencephalogram (EEG). Continuous infusion of pentobarbital, titrated to a burst-suppression EEG pattern, is used for refractory intracranial hypertension as well as status epilepticus. Hypotension and hypopnea frequently accompany the use of all of these drugs, and should be anticipated and aggressively treated. Additionally, an increased risk of ventilator-associated pneumonia has been associated with infusions of pentobarbital, neuromuscular blockade and/or active cooling to maintain euthermia or achieve therapeutic hypothermia. Medical providers should have a high index of suspicion for ventilator-associated infections when using these interventions for TBI [24].

There is little data comparing sedative and analgesic agents in TBI, and medication choice and dosing are left to treating physician discretion. Additionally, very little is known with regard to the effect of neurotransmitter manipulation on injury exacerbation or impaired recovery. Studies suggest that opioid treatment of increased ICP may be modest and short-lived, and opioids are not only implicated in neuroinflammation but are also known to alter developmental myelination in neonatal rodents [25]. However, severe TBI patients are invariably intubated and mechanically ventilated, and frequently have associated systemic injuries or undergo operative procedures necessitating opioid administration. Nonsteroidal analgesics are either insufficient or generally avoided in the face of bleeding risk from platelet inhibition. GABA agonist exposure is associated with delirium, and may exaggerate traumatic cognitive dysfunction. Prolonged activation of GABA receptors can downregulate their cell surface expression, possibly contributing to an environment prone to excitotoxicity [26]. Many institutions, including our own, sparingly use benzodiazepines since their use may impair neuronal recovery following neurologic injury [27]. Finally, there is increasing evidence in animal models of infant neurodevelopment that propofol and related drugs induce neuronal death, creating a risk-benefit conundrum for anesthesia and critical care providers [28]. Despite these and other knowledge gaps, without compelling evidence, there is currently no equipoise to conduct a study where any of these medication classes might be withheld.

In an attempt to address some of these questions, a prospective observational study by Shein et al. was performed at a tertiary care children's hospital to understand the cerebral hemodynamics of fentanyl, mannitol, hypertonic saline, and pentobarbital in elevated ICP after severe TBI [29••]. Medications were administered during intracranial hypertension crises (ICP $\geq 20 \mathrm{mmHg}$ for $>5 \mathrm{~min}$ ), and mean ICP and CPP values were observed every $5 \mathrm{~s}$. Of note, ICP crises thought to be due to mechanical ventilator changes or external 
stimuli were excluded. Overall, ICP was reduced after administration of fentanyl, hypertonic saline, and pentobarbital. Hypertonic saline resulted in the quickest resolution of intracranial hypertension with the added benefit of increased CPP. Fentanyl was associated with reduction in CPP and exhibited the highest treatment failure rate. As a result of this study and a recent review of the literature, hypertonic saline may be considered as the first-line medication for intracranial hypertension due to its quick resolution of intracranial hypertension and favorable cerebral hemodynamic profile [29••, 30]. Interestingly, mannitol was excluded from analysis in the Shein study due to the small number of doses given, and it is not possible to recommend against the use of mannitol in preference of hypertonic saline based on these and other results $[2,31]$.

\section{Temperature Control}

While the avoidance of hyperthermia is crucial to prevent secondary brain injury, therapeutic hypothermia in pediatric TBI has been considered as a therapeutic strategy after promising results were observed in animal models of TBI [32, 33]. Two notable studies by Hutchison et al. and Adelson et al. have attempted to reconcile any benefit of hypothermia in severe pediatric TBI with the associated risks of infection and thrombosis $[34,35]$. Hutchison et al. performed a multicenter, randomized controlled trial of moderate hypothermia, defined as 32 to $33{ }^{\circ} \mathrm{C}$, initiated within $8 \mathrm{~h}$ of injury and continued for $24 \mathrm{~h}$. There was a concern for increased mortality with no evidence of secondary outcome benefits in the hypothermia group compared to the control group [34]. As a result of this study, Adelson et al. questioned if hypothermia could affect outcomes if patients were cooled for longer (48-72 h compared to $24 \mathrm{~h}$ ) and rewarmed more slowly. Ultimately, the study was terminated early for futility, and concluded that longer periods of hypothermia with slow rewarming does not reduce mortality in severe TBI [35]. Most recently, Tasker et al. conducted a metaanalysis of seven randomized controlled trials of therapeutic hypothermia in pediatric TBI, and concluded that "the null hypothesis of no difference between hypothermia and normothermia on mortality cannot be rejected" [36•]. Interestingly, they observed that the study populations were heterogeneous with respect to important factors, and that therapeutic hypothermia continues to be utilized in pediatric TBI despite the lack of a definitive study to support its efficacy. Specifically, there may be potential subsets of pediatric TBI that might benefit, and Tasker cautions that the search for those subsets should not be prematurely abandoned.

\section{Seizure Prophylaxis}

Posttraumatic seizures (PTS) are a well described and major complication after TBI, and may result in harmful consequences including increased metabolic demand, increased cerebral blood flow and intracranial pressure, and excitotoxic neuronal injury and death. Liesemer et al. report a $12 \%$ incidence of PTS in their observational study of 275 pediatric patients, and the highest risk of PTS appears to be in the moderate and severe TBI groups, children $<2$ years of age, and victims of abusive head trauma [37]. Prevention of secondary brain injury from PTS is an important component of management of patients with TBI, and seizure prophylaxis with phenytoin may be considered in patients with TBI [2]. Despite the guidelines, there is a wide variation in seizure prophylaxis among trauma centers ranging from phenobarbital to levetiracetam, which could explain the disparity between PTS prophylaxis and outcomes [38, 39]. While levetiracetam is frequently used for PTS prophylaxis, a recent study by Chung and O'Brien reports a $17.6 \%$ risk of PTS despite levetiracetam prophylaxis in patients with moderate to severe TBI [40 •]. In their study, this prevalence of PTS in patients receiving levetiracetam was higher than the prevalence of PTS in patients receiving fosphenytoin and similar to patients who received no seizure prophylaxis [40 ${ }^{\bullet}$. The use of levetiracetam for PTS prophylaxis is common and appears to be safe, as there is a wide therapeutic margin and little need for measuring drug levels as is required with fosphenytoin or phenobarbital, but further studies are needed to assess its efficacy, specifically in younger patients and those suffering from AHT $[40 \bullet, 41]$. In our institution, fosphenytoin is the preferred prophylactic and treatment agent for PTS. We also routinely employ continuous EEG on any patient who is receiving neuromuscular blockade, pentobarbital infusion, or is at high risk of PTS.

\section{Decompressive Craniectomy}

While controversial, decompressive craniectomy (DC) with or without duraplasty may be considered in the treatment of intracranial hypertension refractory to medical management or for patients at risk of cerebral herniation [2]. A randomized controlled trial by Cooper et al. investigated if bifrontotemporoparietal decompressive craniectomy in adults suffering from severe TBI and intracranial hypertension, failing first tier therapies, improved functional outcome compared to medical management [42]. Adults in the craniectomy group had lower ICP values with fewer medical interventions, shorter duration of mechanical ventilation, and intensive care unit stay, but their outcome, based on the extended Glasgow outcome scale (eGOS) score, was worse compared to patients undergoing standard care [42]. More recently, the RESCUEicp Trial Collaborators evaluated the effect of DC on clinical outcomes 
(based on eGOS) in patients aged 10-65 years with refractory intracranial hypertension (ICP $>25 \mathrm{mmHg}$ for $1-12 \mathrm{~h}$ ) [43]. Patients were randomly assigned to the medical group or the surgical group where the latter underwent a decompressive craniectomy (either large unilateral frontotemporoparietal or bifrontal craniectomy). Overall, patients who underwent DC had $22 \%$ lower mortality albeit with higher rates of vegetative state, lower severe disability, and upper severe disability compared to the medically managed cohort [43]. To date, there have been no dedicated randomized controlled trials evaluating DC in pediatric TBI, and the pediatric evidence for DC is limited to small case series.

\section{Outcomes}

Overall, morbidity and mortality from pediatric TBI is associated with the extent of primary injury and the effectiveness of mitigating secondary injury. The severity of both primary and secondary injuries, in addition to an initial low GCS, is associated with a lower Glasgow outcome scale score and poor outcome [44]. Mortality from severe TBI has been reported as high as $50 \%$, and Sharples et al. reported a fourfold increase in mortality in pediatric patients who suffered from hypotension and hypoxia $[45,46]$. More recently, new data from an initial 200 patient cohort of the Approaches and Decisions in Acute Pediatric TBI (ADAPT) trial found a mortality of $19 \%$ in children with severe TBI, and there was a strong association between GCS score at the time of ICP monitor placement and mortality [47]. Patients with severe TBI are frequently left with neurological sequelae, including but not limited to paresis, cognitive impairment, psychiatric illness, behavioral disorders, epilepsy, and dysphagia requiring a gastrostomy tube, and these deficits may lead to a reduced quality of life $[9,48]$.

Outcomes can be improved with the implementation of specialized teams practicing evidenced-based treatment [49]. Early involvement of pediatric-trained speech, occupational, and physical therapists, as well as physical medicine and rehabilitation providers, is crucial in maximizing neurologic recovery following TBI. Patients often benefit from pediatric rehabilitation programs, either in an inpatient or outpatient setting, depending on the severity of deficits.

\section{Conclusions}

Traumatic brain injury continues to be a major health concern due to its contribution to worldwide morbidity and mortality. Several studies have expanded our knowledge of both the pathophysiology and management of pediatric TBI since the release of the 2012 Traumatic Brain Injury Guidelines. Nevertheless, these guidelines emphasize the continued lack of dedicated pediatric evidence, particularly high quality, randomized controlled trials. We look forward to the results of the ADAPT trial, which is an international comparative effectiveness trial with aims to investigate current practice trends and outcomes of children with severe TBI (adapttrial. org), and we are hopeful the ADAPT trial will result in some of these knowledge gaps being filled as well as directing future investigations in pediatric TBI.

\section{Compliance with Ethical Standards}

Conflict of Interest The authors declare that they have no conflicts of interest.

Human and Animal Rights and Informed Consent This article does not contain any studies with human or animal subjects performed by any of the authors.

\section{References}

Papers of particular interest, published recently, have been highlighted as:

- Of importance

•- Of major importance

1 Anderson V, Moore C. Age at injury as a predictor of outcome following pediatric head injury: a longitudinal perspective. Child Neuropsychol. 1995;1(3):187-202.

2 Kochanek PM, et al. Guidelines for the acute medical management of severe traumatic brain injury in infants, children, and adolescents - second edition. Pediatr Crit Care Med. 2012;13(Suppl 1): S1-82.

3 Taylor CA, et al. Traumatic brain injury-related emergency department visits, hospitalizations, and deaths-United States, 2007 and 2013. MMWR Surveill Summ. 2017;66(9):1-16.

4 Xiang J, et al. Paediatric patients with abusive head trauma treated in US emergency departments, 2006-2009. Brain Inj. 2013;27(1314):1555-61.

5. Miller Ferguson N, et al. Abusive head trauma and mortality-an analysis from an international comparative effectiveness study of children with severe traumatic brain injury. Crit Care Med. 2017;45(8):1398-407. This study reports similar mortality between AHT and accidental TBI with leading cause of death in both being increased ICP, as well as female predominance in the AHT cohort.

6. Chesnut RM, et al. The role of secondary brain injury in determining outcome from severe head injury. J Trauma. 1993;34(2):21622.

7. Vavilala MS, et al. Blood pressure and outcome after severe pediatric traumatic brain injury. J Trauma. 2003;55(6):1039-44.

8. Kannan N, et al. Timely hemodynamic resuscitation and outcomes in severe pediatric traumatic brain injury: preliminary findings. Pediatr Emerg Care. 2016. https://doi.org/10.1097/PEC.0000000000000803

9. Kapapa T, et al. Head trauma in children, part 2: course and discharge with outcome. J Child Neurol. 2010;25(3):274-83.

10. Chambers IR, Treadwell L, Mendelow AD. Determination of threshold levels of cerebral perfusion pressure and intracranial pressure in severe head injury by using receiver-operating characteristic curves: an observational study in 291 patients. J Neurosurg. 2001;94(3):412-6. 
11. Allen BB, et al. Age-specific cerebral perfusion pressure thresholds and survival in children and adolescents with severe traumatic brain injury. Pediatr Crit Care Med. 2014;15(1):62-70. This paper supports the use of age-specific CPP thresholds.

12. Miller Ferguson $\mathrm{N}$, et al. Intracranial hypertension and cerebral hypoperfusion in children with severe traumatic brain injury: thresholds and burden in accidental and abusive insults. Pediatr Crit Care Med. 2016;17(5):444-50.

13. Adelson PD, et al. Phase II clinical trial of moderate hypothermia after severe traumatic brain injury in children. Neurosurgery. 2005;56(4):740-54. discussion 740-54

14. Pfenninger J, Santi A. Severe traumatic brain injury in children - are the results improving? Swiss Med Wkly. 2002;132(9-10):116-20.

15. Chambers IR, et al. Age-related differences in intracranial pressure and cerebral perfusion pressure in the first 6 hours of monitoring after children's head injury: association with outcome. Childs Nerv Syst. 2005;21(3):195-9.

16. Jagannathan J, et al. Long-term outcomes and prognostic factors in pediatric patients with severe traumatic brain injury and elevated intracranial pressure. J Neurosurg Pediatr. 2008;2(4):240-9.

17. Shore PM, et al. Continuous versus intermittent cerebrospinal fluid drainage after severe traumatic brain injury in children: effect on biochemical markers. J Neurotrauma. 2004;21(9):1113-22.

18. Nwachuku EL, et al. Intermittent versus continuous cerebrospinal fluid drainage management in adult severe traumatic brain injury: assessment of intracranial pressure burden. Neurocrit Care. 2014;20(1):49-53.

19. Caruselli $\mathrm{G}$, et al. The role of CSF ventricular drainage in controlling intracranial hypertension in patients with brain lesions. Comparison of three methods. Preliminary results. J Neurosurg Sci. 1992;36(4):219-25.

20. Kochanek PM, et al. The potential for bio-mediators and biomarkers in pediatric traumatic brain injury and neurocritical care. Front Neurol. 2013;4:40.

21. Zeiler FA, et al. The ketamine effect on ICP in traumatic brain injury. Neurocrit Care. 2014;21(1):163-73.

22. Wang $X$, et al. Ketamine does not increase intracranial pressure compared with opioids: meta-analysis of randomized controlled trials. J Anesth. 2014;28(6):821-7.

23. Bar-Joseph G, et al. Effectiveness of ketamine in decreasing intracranial pressure in children with intracranial hypertension. $\mathrm{J}$ Neurosurg Pediatr. 2009;4(1):40-6.

24. Hamele $\mathrm{M}$, et al. Ventilator-associated pneumonia in pediatric traumatic brain injury. J Neurotrauma. 2016;33(9):832-9.

25. Vestal-Laborde AA, et al. The opioid system and brain development: effects of methadone on the oligodendrocyte lineage and the early stages of myelination. Dev Neurosci. 2014;36(5):409-21.

26. Barnes EM Jr. Use-dependent regulation of GABAA receptors. Int Rev Neurobiol. 1996;39:53-76.

27. Goldstein LB. Basic and clinical studies of pharmacologic effects on recovery from brain injury. J Neural Transplant Plast. 1993;4(3): 175-92.

28. Walters JL, Paule MG. Review of preclinical studies on pediatric general anesthesia-induced developmental neurotoxicity. Neurotoxicol Teratol. 2017;60:2-23.

29.• Shein SL, et al. Effectiveness of pharmacological therapies for intracranial hypertension in children with severe traumatic brain injuryresults from an automated data collection system time-synched to drug administration. Pediatr Crit Care Med. 2016;17(3):236-45. This recent article addresses the lack of studies evaluating the efficacy of medication management of increased ICP, showing that hypertonic saline was the most effective in decreasing ICP while also maintaining/improving CPP.

30. Alnemari AM, et al. A comparison of pharmacologic therapeutic agents used for the reduction of intracranial pressure following traumatic brain injury. World Neurosurg. 2017;13(17):1878-8750.
31. Shein SL, et al. Effectiveness of pharmacological therapies for intracranial hypertension in children with severe traumatic brain injury-results from an automated data collection system timesynched to drug administration. Pediatr Crit Care Med. 2016;17(3):236-45.

32. Clark RS, et al. Mild posttraumatic hypothermia reduces mortality after severe controlled cortical impact in rats. J Cereb Blood Flow Metab. 1996;16(2):253-61.

33. Clifton GL, et al. Marked protection by moderate hypothermia after experimental traumatic brain injury. J Cereb Blood Flow Metab. 1991;11(1):114-21.

34. Hutchison JS, et al. Hypothermia therapy after traumatic brain injury in children. N Engl J Med. 2008;358(23):2447-56.

35. Adelson PD, et al. Comparison of hypothermia and normothermia after severe traumatic brain injury in children (Cool Kids): a phase 3, randomised controlled trial. Lancet Neurol. 2013;12(6):546-53.

36. Tasker RC, et al. Updating evidence for using hypothermia in pediatric severe traumatic brain injury: conventional and Bayesian meta-analytic perspectives. Pediatr Crit Care Med. 2017;18(4):355-62. Meta-analysis of all hypothermia studies in pediatric TBI, warning to not completely dismiss therapeutic hypothermia, that there may be still be a target population

37. Liesemer K, et al. Early post-traumatic seizures in moderate to severe pediatric traumatic brain injury: rates, risk factors, and clinical features. J Neurotrauma. 2011;28(5):755-62.

38. Kurz JE, et al. Variation in anticonvulsant selection and electroencephalographic monitoring following severe traumatic brain injury in children - understanding resource availability in sites participating in a comparative effectiveness study. Pediatr Crit Care Med. 2016;17(7):649-57.

39. Ostahowski PJ, et al. Variation in seizure prophylaxis in severe pediatric traumatic brain injury. J Neurosurg Pediatr. 2016;18(4):499-506.

40. Chung MG, O'Brien NF. Prevalence of early posttraumatic seizures in children with moderate to severe traumatic brain injury despite levetiracetam prophylaxis. Pediatr Crit Care Med. 2016;17(2):150-6. This study questions the efficacy of the use of levetiracetam in prophylaxis for posttraumatic seizures, which has become widespread.

41. Pearl PL, et al. Results of phase II levetiracetam trial following acute head injury in children at risk for posttraumatic epilepsy. Epilepsia. 2013;54(9):e135-7.

42. Cooper DJ, et al. Decompressive craniectomy in diffuse traumatic brain injury. N Engl J Med. 2011;364(16):1493-502.

43. Hutchinson PJ, et al. Trial of decompressive craniectomy for traumatic intracranial hypertension. N Engl J Med. 2016;375(12): 1119-30.

44. Chiaretti A, et al. Prognostic factors and outcome of children with severe head injury: an 8-year experience. Childs Nerv Syst. 2002;18(3-4):129-36.

45. Adelson PD, Kochanek PM. Head injury in children. J Child Neurol. 1998;13(1):2-15.

46. Sharples PM, et al. Avoidable factors contributing to death of children with head injury. BMJ. 1990;300(6717):87-91.

47. Murphy S, et al. Tripartite stratification of the Glasgow Coma Scale in children with severe traumatic brain injury and mortality: an analysis from a multi-center comparative effectiveness study. J Neurotrauma. 2017. https://doi.org/10.1089/neu.2016.4793

48. Kapapa T, et al. Head trauma in children, part 3: clinical and psychosocial outcome after head trauma in children. J Child Neurol. 2010;25(4):409-22.

49. Pineda JA, et al. Effect of implementation of a paediatric neurocritical care programme on outcomes after severe traumatic brain injury: a retrospective cohort study. Lancet Neurol. 2013;12(1):45-52. 